\title{
High-temperature series expansion of the magnetic susceptibility of extended magnetic systems in a complete computer implementation
}

\author{
Th. Eifert ${ }^{\text {a }}$, F. Hüning ${ }^{\mathrm{b}}$, H. Lueken ${ }^{\mathrm{b}, *}$ P. Schmidt ${ }^{\mathrm{c}}$, G. Thiele $^{\mathrm{c}}$ \\ ${ }^{a}$ Rechenzentrum der RWTH Aachen, Seffenter Weg 23, 52074 Aachen \\ ${ }^{\mathrm{b}}$ Institut für Anorganische Chemie, Prof.-Pirlet-Str. 1, 52074 Aachen \\ ${ }^{\mathrm{c}}$ Institut für Anorganische und Analytische Chemie der Albert-Ludwigs-Universität \\ Freiburg, Albertstr. 21, 79104 Freiburg i. Br.
}

In memoriam of Professor Louis Néel who passed away on November 17, 2000 at age 96

\begin{abstract}
The high-temperature series expansion (HTSE) of the susceptibility of Heisenberg magnets is formulated completely in algorithms and implemented in computer programs for the first time. Specifying the system under investigation with respect to the spin $S\left(\frac{1}{2}, 1, \ldots, \frac{7}{2}\right)$ of the magnetic centres, their partial structure and their neighbourhood relationship (first, second and third neighbours), the susceptibility equation is automatically developed up to the required order as a function of the exchange parameters $J_{1}, J_{2}$, and $J_{3}$. The calculations involve drawing of the relevant interaction graphs and computation of the trace of the corresponding spin products as well as the determination of the lattice count. The performance of the HTSE program is demonstrated by analysing the magnetic properties of $\mathrm{CrBr}_{3}$ for $T>T_{C}$.
\end{abstract}

* Corresponding author.

Email addresses: eifert@rz.rwth-aachen.de (Th. Eifert),

Felix.Huening@infineon.com (F. Hüning), heiko.lueken@ac.rwth-aachen.de

(H. Lueken), (P. Schmidt), (G. Thiele). 


\section{Introduction}

In view of the great interest in the synthesis of new magnetic materials, e.g. molecular magnets, and their precise magnetochemical characterization with the present generation of SQUID magnetometers, an adequate tool is needed in extracting the relevant exchange (spin-spin) coupling parameters $J_{i}$ from experimental susceptibility data. With regard to the region above the critical point $T_{C}$ (Curie temperature) or $T_{N}$ (Néel temperature), high-temperature series expansions (HTSE), developed by Rushbrooke and Wood [1] for nearest neighbours (n) and extended by Wojtowicz and Joseph [2] for next-nearest ones (nn), play a dominant role on account of general applicability for ferromagnetic as well as antiferromagnetic interactions [3,4]. While series expansions are already available for several ideal systems of high symmetry and reduced lattice dimensionality (e.g. simple cubic, square planar, honeycomb, Kagomé etc., often for restricted spin $S$ and n neighbours only [5,6]), a computer program of general applicability with respect to spin, lattice and neighbourhood relationship as well as to the order of the series expansion is still lacking.

In the following the complete computer implementation, applicable to real magnetic systems with equivalent centres, is described which allows the calculation of the zero-field magnetic susceptibility of exchange-coupled Heisenberg systems in general. One is free to choose (i) the magnetic centre, i.e. $S=\frac{1}{2}, 1, \ldots, \frac{7}{2}$, (ii) the centres' spatial arrangement, (iii) the assignment of neighbours, i. e. n, nn, nnn, and (iv) the order of the series expansion.

\section{Fundamental principles of HTSE: General susceptibility equa- tion for $n, n n$, and nnn neighbour interactions}

The hamiltonian for an isotropic exchange coupled system in a static magnetic field is represented by $[7,3]$

$$
\hat{H}=-2 \sum_{<i, j>} J_{i j} \hat{\boldsymbol{S}}_{i} \cdot \hat{\boldsymbol{S}}_{j}-g \mu_{B} B_{z} \sum_{p} \hat{S}_{p z}
$$

with exchange parameters $J_{i j}>0$ in the case of ferromagnetic and $<0$ in the case of antiferromagnetic coupling. The suffices $i, j$ refer to the sites of a crystal lattice, $\langle i, j\rangle$ denotes a pair of sites, each such pair being counted once only. $\hat{\boldsymbol{S}}_{i}$ and $\hat{S}_{i z}$ is the three-dimensional spin operator and its $z$ component, respectively, referring to the $i$ th center, $g$ is the Landé factor, and $\mu_{B}$ the Bohr magneton, $B_{z}$ is the magnetic induction of the external field in $z$ direction.

For any matrix representation of $\hat{H}$ the partition function $Z=\operatorname{Tr}[\exp (-\beta \hat{H})]$ with $\beta \equiv 1 /\left(k_{B} T\right)$ mediates between $\hat{H}$ and the experimentally available quantities, e.g. the magnetic susceptibility where $T$ is the absolute temperature and 
$k_{B}$ the Boltzmann constant. From statistical thermodynamics the magnetic susceptibility per mol $\chi_{m o l}$ follows as

$$
\chi_{m o l}=\mu_{0} N_{A} k_{B} T \frac{\partial^{2}}{\partial B^{2}} \ln Z=\mu_{0} \frac{\partial M_{m o l}}{\partial B},
$$

where $\mu_{0}$ is the magnetic permittivity, and $N_{A}$ the Avogadro number.

In the HTSE the partition function $Z$ is expanded around $\beta=0$ corresponding to the Taylor series of the exponential function. Finally, the susceptibility per mol with three exchange parameters $J_{1}, J_{2}$ and $J_{3}$ for $\mathrm{n}$, nn and nnn neighbours, respectively, in the zero field limit is given by

$$
\chi_{m o l}(B \rightarrow 0)=\mu_{0} \frac{N_{A} g^{2} \mu_{B}^{2}}{k_{B} T} \frac{\operatorname{Tr}\left[\hat{Q}^{2} \sum_{k} \sum_{l=0}^{k} \sum_{\substack{m=0 \\ n=k-l-m}}^{k-l} \frac{(2 \beta)^{k}}{k !} J_{1}^{l} J_{2}^{m} J_{3}^{n} \hat{\boldsymbol{P}}^{k}\right]}{\operatorname{Tr}\left[\sum_{k} \sum_{l=0}^{k} \sum_{\substack{m=0 \\ n=k-l-m}}^{k-l} \frac{(2 \beta)^{k}}{k !} J_{1}^{l} J_{2}^{m} J_{3}^{n} \hat{\boldsymbol{P}}^{k}\right]}
$$

with

$$
\hat{\boldsymbol{P}}=\sum_{<i, j>} \hat{\boldsymbol{S}}_{i} \cdot \hat{\boldsymbol{S}}_{j} \quad \text { and } \quad \hat{Q}^{2}=\sum_{i} \hat{S}_{i z} \sum_{j} \hat{S}_{j z}
$$

where $\hat{\boldsymbol{P}}$ is the spin product that represents the coupling between two magnetic centres, and $\hat{Q}^{2}$ is the product of the $z$-component of these spin matrices of $\hat{\boldsymbol{P}}$.

The way we deduced eq. (3) (and thus the equation itself) is quite straightforward, in contrast to former works (e. g. [2]) where computational issues of that time forced to deviate from this way.

\section{Algorithms and computer implementation}

The HTSE is valid for $T>T_{C}\left(T_{N}\right)$ in the zero field limit. The gradual change from only single-centre effects at high temperatures (i.e., $\beta=0$ ) to more and more extended regions of local coupling when approaching $T_{C}\left(T_{N}\right)$, is reflected in the increasing number of terms necessary for the HTSE.

To develop eq. (3) for the lattice under study the contributing terms to each spin product and their frequency have to be determined $[1,3,2]$. For this purpose, the spin product of order $k, \hat{\boldsymbol{P}}^{k}$, is taken as a graph consisting of nodes and $k$ lines where each node corresponds to a magnetic centre and each line represents a neighbourhood relationship. The computation of the contributing terms to eq. (3) thus falls into three separate tasks:

- to generate only once all possible graphs of $k$ lines; 
- to compute the trace of the spin product that belongs to each graph;

- to obtain the lattice count (LC), i. e. the number of how often each graph can be placed onto a given lattice.

While the first two tasks are lattice independent, the latter builds the correlation with the lattice under investigation and the number of different bonds (n, nn, nnn). Our work focused on the first and third task while we implemented the second task mainly as described in refs. [1,3].

\subsection{List of graphs}

The number of individual graphs that must be taken into account grows fast with the order of the series expansion. For the generation of all possible graphs that may evolve from the series expansion of the partition function $Z$, we found no systematic approach in literature, just graphs collected in tabular form [8]. In our approach, the generation of graphs is done by a recursive algorithm. To generate graphs of $k$ lines from a specific graph with $k-1$ lines, a new line is added in sequence to every node of the latter or to a new node. This line ends either on an already present node or on a new one.

This approach does generate the complete list of graphs of specific $k$, but produces most of them more than once. To avoid the creation of duplicates, the set of starting and ending nodes of a graph is limited to the last two generated nodes or a new node. Finally, before entering the list, each graph in form of the corresponding adjacency matrix is matched for equivalency to each other and rejected if necessary. Thus, the list contains every possible graph exactly once, in consistence with manually derived lists. However, not all of these play a role in the development of eq. (3) on account of vanishing trace and compensation. This is automatically taken into consideration in the second task (determination of spin products [1,3]) and subsequent steps, respectively. Here also the term $\hat{Q}^{2}$ is taken into account where the $S_{i z}$ and $S_{j z}$ are placed at all nodes of the graph and thus yield multiple different spin products.

\subsection{Determination of the lattice count (LC)}

The computations until here are independent of a particular lattice. The correlation between the spin products and the lattice is expressed by the LC. For some of the common lattices (square, trigonal, s.c., b.c.c., and f.c.c.) the LCs for all of the 1457 basic graphs, i. e. graphs with single lines only, out of up to ten lines are tabulated [8], but only for nearest-neighbour interactions. A systematic approach for the determination of the LC for other lattices and, in particular, for different neighbours, was not published before. 


\subsubsection{Simple graphs}

Our algorithm uses the fact that in the scope of HTSE all magnetic centres are equivalent. We thus take one centre on the lattice as reference atom, i. e., the starting atom for our subsequent considerations. For any mapping we place the first node of the graph onto this atom. This first node may be any of the nodes; they are just worked through in sequence. The next node of the graph, i. e. the ending point of the current line, is then placed on a centre that occurs in the reference centre's adjacency list. By this we have mapped the first line of the graph between the first pair of neighbours on the lattice. From this second node-centre pair with its associated adjacency list, we do the analogous step for the next line until we have recursively mapped the complete graph, so here the graph which we are counting the LC for has found its first complete mapping. The algorithm then takes back one line of the graph and maps the endpoint of this line on the next centre in the adjacency list, so the 'tail' of the graph gets placed on all of the neighbours of the next-to-last atom. This is continued until the whole tree of recursive mappings is walked through. At this moment we've found all possible mappings with the first node of the graph being mapped onto the reference atom mentioned above. Anyway, the mapping is not yet complete. To get there, all the nodes of the graph are subsequently used as starting points for the recursive algorithm explained above. For runtime reasons we do not re-run the tree for topologically equivalent nodes of the graph.

By now we have accumulated a list of graphs of all possible mappings, but as in the case of graph generating, there are duplicates in the list that are eliminated by the same routines as before.

\subsubsection{Multipart Graphs}

As explained in [1], disconnected graphs do contribute only if their parts have atoms in common (i. e. are re-connected), so these parts have to be investigated whether or not this condition is met.

The treatment of such disconnected graphs has been discussed by Domb [5,9], but the algorithm used there could not be transferred to computers. Anyway, his results for the lattices he worked on served as a reference for us. We've been able to verify all except two of the LC's given there: for the simple cubic lattice the LC of 146 for graph (12)(34)(56) (with $(i j)$ denoting the line connecting centre $i$ with centre $j$ ) must be replaced by the value of 110.5, and the LC of 3753 for graph (12)(23)(45)(67) must read 2946. While checking these values we came across a proximate pitfall which leads to the erroneous values.

This case of multipart graphs is handled by an additional nest of loops where every separate part of the graph is mapped onto the lattice, using each of the centers as a starting point that the previous 'piece' of the graph is mapped onto. In other words, the first of the multiple parts is treated as before. For the second part, each of the atoms where a node of the first part is mapped onto is taken as the reference atom, so that this second part starts from each 
of the atoms of the first part. Starting from each of these reference atoms, the second part of the graph is mapped onto the lattice as described before. We then have generated all possible mappings of the two parts onto the lattice where at least the reference atom is common to both of the parts. If there are more than two parts, this is repeated, with the new reference atom being each of the atoms of the combined graph in sequence.

\subsection{Different neighbours}

Inspecting eq. (3), we see that the coupling parameters $J_{1}, J_{2}$, and $J_{3}$ for $\mathrm{n}$, nn, and nnn neighbours, respectively, are separated from $\hat{\boldsymbol{P}}^{k}$ which represents the spin product and the corresponding LC. Our approach is to keep this separation, thus having the same graphs and, in particular, the same spin products as before. Consequently, the information about the powers of the different $J$ parameters is obtained by a specific LC for each triple of coupling parameters.

For this purpose, the mapping of a graph onto the lattice keeps track not only of the respective degree of neighbourhood represented by each line, but also of the number of lines, i. e., the multiplicity of the corresponding spin product, to assign this particular LC to the correct $J$-triple. This implies that, in contrast to nearest-neighbour algorithms, graphs of the same shape, but with different multiplicities of lines can no longer be treated as the same. Instead, these are all analysed separately. Consequently, such mappings of the graph that yield different $J$-triples are counted separately $[10,11]$.

The lattice in Fig. 1 is an example which embodies all the points we discussed so far. This system needs three different neighbour relationships to be described where our algorithm is the first to systematically compute the LC for a lattice with different couplings having the same spatial orientation [12].

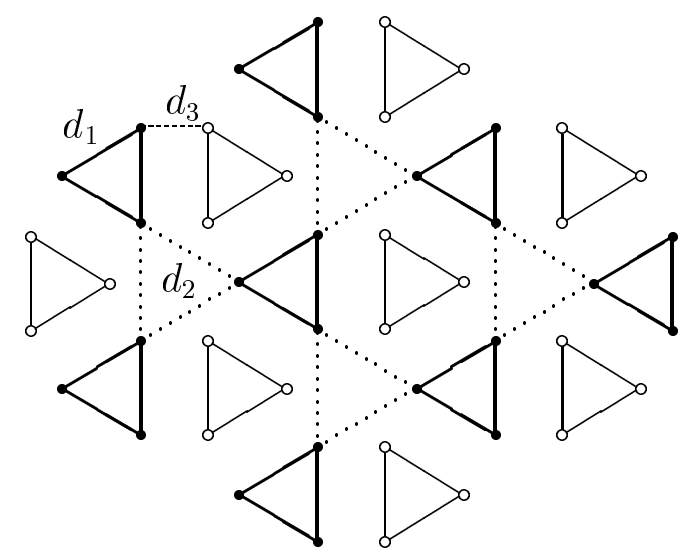

Fig. 1: Eu partial structure in $\mathrm{EuMg}_{5.2}$ [?] with topologically equivalent triangles of different size $(\bullet$ and $\circ$ mark different layers) 


\section{Application to $\mathrm{CrBr}_{3}$}

The essentially perfect Heisenberg ferromagnet $\mathrm{CrBr}_{3}\left(T_{C}=32.5 \mathrm{~K} ; \mathrm{BiI}_{3^{-}}\right.$ type layer structure, space group $R \overline{3}, a_{\text {hex }}=630.7 \mathrm{pm}, c_{\text {hex }}=1838.3 \mathrm{pm}$; hcp arrangement of $\mathrm{Br}, \mathrm{Cr}$ in octahedral holes forming $6^{3}$ nets, see Fig. 4) has been chosen to demonstrate the performance of the HTSE-package especially since exchange parameter values for up to fifth-nearest neighbours are available on the basis of a spin wave study by inelastic neutron scattering [13].

Samples of high purity were prepared by chemical vapour transport [14]. Magnetic properties have been determined by SQUID magnetometry (MPMS-5S, Quantum Design) in the temperature range $1.7-400 \mathrm{~K}$ at applied fields in the range $0.01-5 \mathrm{~T}$. Applying the HTSE-package program the susceptibility expression was developed up to sixth order in a period of $10 \mathrm{~d}$ (SGI Origin computer) as a function of $g$ and three exchange parameters $J_{i}(i=1,2,3)$. $J_{1}$ and $J_{3}$ stand for the intralayer interactions, the former referring to the three neighbours $\mathrm{n}$ at $d_{1}=364 \mathrm{pm}$ and the latter to the six neighbours nnn at $d_{3}$ $=631 \mathrm{pm} . J_{2}$ applies to the single neighbour nn at $d_{2}=612 \mathrm{pm}$ placed within one adjacent layer, while nine further interlayer neighbours, not considered in the HTSE, are $713 \mathrm{pm}$ apart. $J_{1}$ and $J_{2}$ are expected to be positive on account of the overall ferromagnetic behaviour where $J_{1}$ is distinctly larger than $J_{2}$ according to the superexchange pathways via cation-anion-cation and cation-anion-anion-cation, respectively.

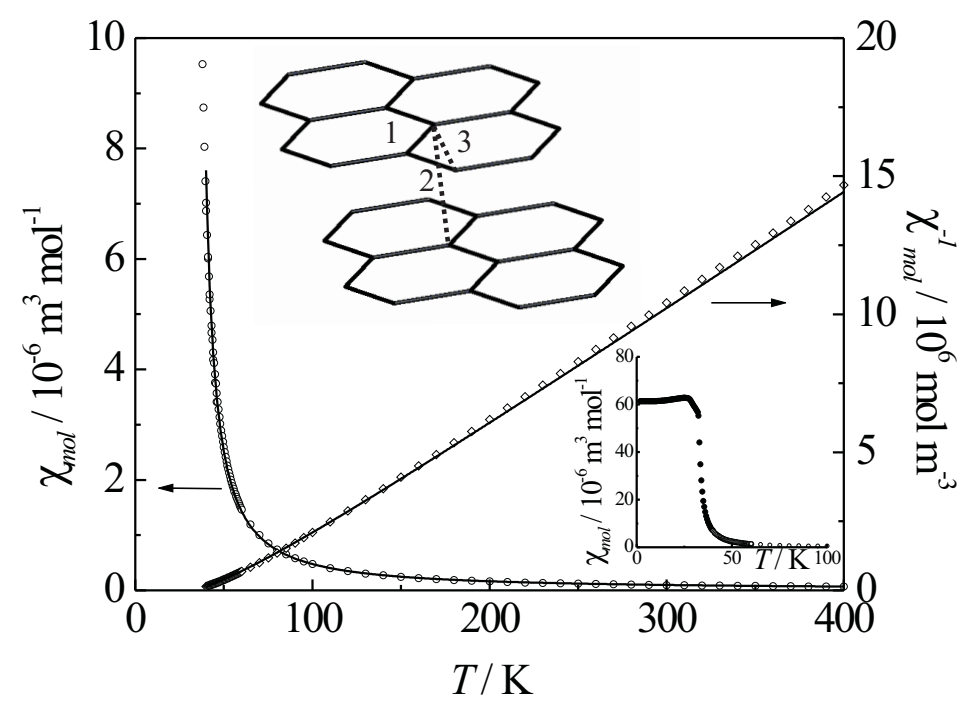

Abb. 4: $\mathrm{Cr}^{3+}(S=3 / 2)$ partial structure in $\mathrm{CrBr}_{3}$ and $\chi_{m o l}$ and $\chi_{m o l}^{-1}$ as a function of temperature (applied field: $B_{0}=0.1 \mathrm{~T}$ ); the solid lines refer to the HTSE model fit 2 (see text) with $g=1.953, J_{1}=6.885 \mathrm{~cm}^{-1}, J_{2}=0 \mathrm{~cm}^{-1}$ (fixed), $J_{3}=$ $-0.082 \mathrm{~cm}^{-1}$ (quality of the fit: $S Q=0.9 \% ; S Q=(F Q / n)^{1 / 2} \times 100 \%$, with $F Q=$ $\left.\sum_{i=1}^{n}\left\{\left[\chi_{o b s}(i)-\chi_{c a l}(i)\right] / \chi_{o b s}(i)\right\}^{1 / 2}\right)$ 
In fitting procedures $\left.{ }^{1}\right)$ an adaptation of high quality $(S Q=0.9 \%$, see caption to Fig. 4) is already produced with the parameter values $g=1.953$ close to the expected value for a pure spin system and only the two intralayer interaction parameters $J_{1}=6.885 \mathrm{~cm}^{-1}$ and $J_{3}=-0.082 \mathrm{~cm}^{-1}$. This result speaks for an intralayer ferromagnetic coupling dominated by the nearest-neighbour interaction and rather weak interlayer interactions. Although $J_{3}$ is only a small quantity it is essential for a satisfactory fit. Keeping $J_{3}=0$ worsens the fit considerably $(S Q \approx 12 \%)$. Worth mentioning is the very good fit by refining the pair $J_{1}, J_{2}$. However, since a negative $J_{2}$ is obtained in disagreement with the observed spontaneous magnetization at low temperature this result is ruled out.

Including the interlayer parameter $J_{2}$ in the refinements, starting from the high quality fit so far achieved with optimized $g, J_{1}$, and $J_{3}$, gives no improvement. Even the consideration of further measurement data closer to $T_{C}$, at the cost of the quality of the fits, are not successful. Thus, the magnitude of $J_{2}$ can not be reliably extracted from the susceptibility data without further assumptions. Allowing, on the one side, for $0<J_{2}<0.1 J_{1}$ in a series of computations, where the higher boundary seems to be a pragmatical value with regard to the different intra- and interlayer superexchange pathways [15], lead to simulations of identical high quality. Increasing $J_{2}\left(0 \rightarrow 0.6 \mathrm{~cm}^{-1}\right), g$ and $J_{1}$ remain unaffected, while the absolute value of $J_{3}$ increases $\left(-0.082 \rightarrow-0.190 \mathrm{~cm}^{-1}\right)$. On the other side, the results of the spin-wave analysis $J_{1}=0.849 \mathrm{meV}=$ $6.85 \mathrm{~cm}^{-1}, J_{3}=-0.0143 \mathrm{meV}=-0.115 \mathrm{~cm}^{-1}$ as well as rather weak interlayer couplings owing to competing ferromagnetic and antiferromagnetic interactions [13] agree remarkably with our findings. Keeping in the HTSE analysis the ratio $J_{3} / J_{1}$ constant at -0.0168 which follows from the spin wave analysis, yields $J_{2}=0.162 \mathrm{~cm}^{-1}$ which lies in the above mentioned reasonable range.

To conclude, by magnetochemical analysis of the precise susceptibility data of $\mathrm{CrBr}_{3}$ the dominant intralayer exchange interaction, represented by $J_{1}$, has been reliably determined, and it is demonstrated that a small antiferromagnetic intralayer component $J_{3}$ is essential in $\mathrm{CrBr}_{3}$. Attempts to determine reliably the interlayer parameter $J_{2}$ by inclusion of measurement data closer to $T_{C}$ failed. In this respect an increase of the order $>6$ of the HTSE is necessary.

\section{Conclusion and perspective}

We showed how the computation of the general susceptibility eq. (3) can be split into three separate tasks ((i) graphs corresponding to spin products, (ii) traces of spin products, (iii) lattice counts) and how these are solved by our

$\overline{1}$ ) To improve the convergence behaviour of the HTSE, various Padé approximations $[M / L]$ with $M+L=6$ have been tested where [3/3] turned out to be best. 
implementation. The extension of the depicted algorithm to nn and nnn neighbours did increase dramatically the algorithmic and thus the computational effort, but enables the fully automated computation of the HTSE which otherwise could not be treated adequately, e.g. the modified Kagomé lattice of divalent europium in $\mathrm{EuMg}_{5.2}[10]$ and the stacked honeycombs of trivalent chromium in $\mathrm{CrBr}_{3}$. In addition, the programs are about to be parallelized to exploit current computer hardware and thus to enable us to tackle significantly larger problems, e.g. orders $>6$ of the expansions. On the long term, the computational part might be extended to handle anisotropic coupling as well.

Under the present conditions the development of orders $>6$ is excluded on account of time consumption.

\section{References}

[1] G. S. Rushbrooke, P. J. Wood, Mol. Phys. 1 (1958) 257.

[2] P. J. Wojtowicz, R. I. Joseph, Phys. Rev. A 161 (1964) 1314.

[3] G. S. Rushbrooke, G. A. Baker, P. J. Wood, in: C. Domb, M. S. Green (Eds.), Phase Transitions and Critical Phenomena, Vol. 3, Chap. 5; Academic Press, London · New York, 1974, p. 245.

[4] R. Navarro, in: L. J. de Jongh (Ed.), Magnetic Properties of Layered Transition Metal Compounds, Kluwer, Dordrecht · Boston · London, 1990, p. 105.

[5] C. Domb, Adv. Phys. 9 (1960) 149 and 245.

[6] C. Domb, in: C. Domb, M. S. Green (Eds.), Phase Transitions and Critical Phenomena, Vol. 3, Chap. 6; Academic Press, London · New York, 1974, p. 357.

[7] J. H. Van Vleck, The Theory of Electric and Magnetic Susceptibilities, Oxford University Press, Oxford, 1932.

[8] G. A. Baker, Jr., H. E. Gilbert, J. Eve, G. S. Rushbrooke, A Data Compendium of Linear Graphs with Application to the Heisenberg Model, Brookhaven National Laboratory Report No. BNL 50053 (1967)

[9] C. Domb, M. F. Sykes, The Calculation of Lattice Constants in Crystal Statistics, Phil. Mag. 2 (1957) 733

[10] Th. Eifert, Thesis, RWTH Aachen (1999)

[11] Th. Eifert, Software 'HTSE Package', RWTH Aachen (2001), http://www.rz.rwth-aachen.de/eifert/information/HTSE

[12] J. L. Martin in: C. Domb, M. S. Green (Eds.), Phase Transitions and Critical Phenomena, Vol. 3, Chap. 5; Academic Press, London · New York, 1974, p. 97. 
[13] E. J. Samuelsen, R. Silberglitt, G. Shirane, J. P. Remeika, Phys. Rev. B 3 (1971) 157

[14] P. Schmidt, Thesis, Universität Freiburg i. Br. (1999)

[15] J. B. Goodenough, Magnetism and the Chemical Bond, Interscience, New York, London 1963 